\title{
Postpartum sterilization choices made by HIV-infected women
}

\author{
GRETCHEN S. STUART, PAULA M. CASTAÑO, JEANNE S. SHEFFIELD, \\ BARBARA McELWEE, DONALD D. McINTIRE, \& GEORGE D. WENDEL \\ Department of Obstetrics \& Gynecology, The University of Texas Southwestern Medical Center, Dallas, Texas, USA
}

\begin{abstract}
Objective. To assess if HIV-infected women made different choices for postpartum sterilization after implementation of the Pediatric AIDS Clinical Trials Group protocol 076 (November 1, 1994) compared to before implementation.

Study design. A retrospective cohort study in which medical records were reviewed to obtain demographic, obstetric and HIV-related data from January 1993 through December 2002. HIV-infected women who completed a pregnancy by birth or abortion were divided into two comparison groups: "Pre-076" and "Post-076". The primary outcome was sterilization by postpartum tubal ligation.

Results. Forty-two women (74\%) in the Pre-076 group chose sterilization compared to 139 of 310 women (45\%) in the Post-076 group (unadjusted OR 3.44, 95\% CI 1.83, 6.47). Seventy-one percent of women younger than 21 years of age in the Pre-076 Group chose sterilization compared with only $35 \%$ of women younger than 21 years in the Post-076 group $(p=0.0136)$. Similarly, $78 \%$ of primiparous women chose sterilization after their first pregnancy in the Pre-076 group, compared to $14 \%$ in the Post-076 group $(p<0.001)$.

Conclusions. Since the implementation of PACTG 076 protocol in November 1994, fewer HIV-infected women chose postpartum sterilization. The typical woman who now chooses postpartum sterilization is less likely to be young or primiparous than those who chose sterilization before PACTG Protocol 076 implementation.
\end{abstract}

Keywords: Postpartum sterilization, reproductive choices, contraception, HIV, PACTG Protocol 076

\section{Introduction}

Over 85,000 women of reproductive age (15-44 years) in the United States are infected with the human immunodeficiency virus (HIV) [1]. Antiretroviral therapy and advances in management of HIV disease have decreased the maternal-infant vertical transmission rate of HIV from $25 \%$ to less than $2 \%$ [2-4], and have changed HIV infection from a life-threatening illness to a chronic disease $[5,6]$. Previous studies have evaluated the impact that knowledge of HIV seropositivity has on the reproductive health decisions made by women [7-10], and HIV-infected women have been reported to be more likely to choose sterilization than women who are not infected [11,12]. In November 1994 the results of the Pediatric AIDS Clinical Trial Group (PACTG) Protocol 076 were published which indicated that antepartum, intrapartum, and neonatal administration of zidovudine could decrease the vertical transmission rate of HIV from $25 \%$ to $8 \%$ [3]. Our study objective was to determine if the postpartum sterilization rate in HIV-infected women changed after implementation of PACTG Protocol 076. A secondary objective was to determine any overall changes in choice of postpartum contraceptive method over the same time period in this population.

\section{Materials and methods}

This was a time-series cohort study at Parkland Memorial Hospital (PMH) from January 1, 1993 through December 31, 2002. PMH is the tertiary care hospital of the Parkland Health and Hospital System which serves Dallas County. PMH has a busy obstetrics service with over 16,000 deliveries a year.

Correspondence: Gretchen S. Stuart, MD, Department of Obstetrics \& Gynecology, The University of Texas Southwestern Medical Center, 5323 Harry Hines Boulevard, Dallas, TX 75390-9032, USA. Tel: 2146487949. Fax: 214648 8499. E-mail: gretchen.stuart@utsouthwestern.edu

Précis

Fewer Human Immunodeficiency Virus infected women chose post-partum sterilization for their contraceptive method after the implementation of Pediatric AIDS Clinical Trials Group Protocol 076 in 1994. 
Prenatal clinics for women with complications of pregnancy are staffed by attending physician faculty. All HIV-infected pregnant women are followed in one multidisciplinary infectious disease obstetrics clinic, where patients are seen by resident physicians and mid-level health care providers. The annual number of HIV-infected pregnant women managed per year at $\mathrm{PMH}$ has ranged from around 40 to 81 during the years studied.

HIV-infected pregnant women are counseled at their first prenatal visit by Maternal Fetal Medicine attendings and one registered nurse. For subsequent visits written protocols are followed when appropriate, and overall supervision is provided by the same faculty physicians who attend the clinic each week. These faculty members coordinate care with Internal Medicine Infectious Disease specialists as necessary. Future plans for birth control are discussed with patients during their prenatal visits and again when they return for their postpartum visits. Women are counseled in a non-judgmental manner, by the provider seeing them for the visit, regarding all contraceptive methods available to them, including the risks and benefits of each method as known at the time. Postpartum sterilization by bilateral tubal ligation (BTL) is one of the options included in those discussions; that option has never been the sole recommendation. The only contraceptive method that has always been recommended is use of condoms.

We included HIV-infected pregnant women who completed a pregnancy during the study period at $\mathrm{PMH}$ and whose complete medical records were available in the study cohort. We divided the complete cohort into a "Pre-076 Group" and a "Post-076 Group" for comparison. The Pre-076 Group comprised HIV-infected pregnant women who did not receive PACTG Protocol 076 counseling because their date of presentation was before November 1994. This counseling included the information that use of zidovudine (ZDV) during the antepartum, intrapartum and neonatal period could decrease the risk of HIV transmission to their infant from $25 \%$ to $8 \%$. The Post-076 Group comprised women who presented after November 1994, or who presented at an early enough gestational age (prior to 14 weeks) to initiate antiretroviral treatment in November 1994. For those HIVinfected women who completed more than one pregnancy at $\mathrm{PMH}$ during the study time period only the decision and pregnancy outcome of the lattermost pregnancy was included in the data analysis. Women were excluded if they had incomplete medical records or pregnancy outcomes we felt would significantly alter sterilization decisions such as fetal demise or fetal death. Maternal death and abortion-hysterectomies were also excluded.
We reviewed medical records and abstracted data for demographic characteristics, delivery or abortion information, AIDS diagnosis according to the Centers for Disease Control (CDC) definition [13], prenatal care attendance, and the choice for postpartum sterilization or other contraceptive method. This was a retrospective chart review of HIV-infected women's choices of postpartum BTL at PMH. We reviewed all data available to us, and therefore no power analysis was done.

Statistical analyses included Pearson Chi-square, and Student's $t$-test for comparison of means. Welch's approximation was applied when necessary to account for unequal variances. The Wilcoxon rank-sum test was used for non-parametric analysis. P-values less than 0.05 were statistically significant. This research was exempted from full review by the Institutional Review Board of the University of Texas Southwestern Medical Center.

\section{Results}

From January 11993 through December 31 2002, 426 HIV-infected women completed at least one pregnancy at Parkland Memorial Hospital. We excluded a total of 59 women; 52 women with incomplete medical records, and seven women with adverse pregnancy outcomes which included 2 women who had fetal demises, one maternal death, three abortion-hysterectomies and one termination for a fetal anomaly. The final cohort consisted of 367 women $(86 \%)$. For the seventy-eight women who completed more than one pregnancy we analyzed only the lattermost pregnancy and the decision made at that pregnancy.

The Pre-076 Group comprised HIV-infected pregnant women who did not receive PACTG Protocol 076 counseling because their date of presentation was before November 1994, the date the manuscript reporting the results of the PACTG 076 study was published [3]. It is likely that results of PACTG 076 were preliminarily available prior to publication and therefore some women prior to this date may have received more extensive counseling regarding the benefits of the triple therapy protocol using antepartum, intrapartum and neonatal zidovudine administration. The decision to choose November 1993, the date of publication of 076, was made because it was an identifiable date. Additionally, with publication comes validation of the results and therefore, acceptance by patients. While this date choice may have been speculative, it was a date which could be pointed to at which counseling would have definitely changed.

Using those criteria, 57 women made up the Pre076 group. The Post-076 Group comprised women who presented after November 1994, or who 
presented at an early enough gestational age (prior to 14 weeks) to initiate anti-retroviral treatment in November 1994. The Post-076 group included 310 women.

The mean age did not differ between the two groups (Table I). There was also no difference in race, with the majority of women being AfricanAmerican, followed by Hispanic and Caucasian. Five percent of the Post-076 group versus $9 \%$ of the Pre-076 group had an HIV-infected child prior to the pregnancy evaluated for the study. While there is not a statistically significant difference between these, the rate of having a previous child infected with HIV was almost doubled in the Post-076 cohort. The majority of women in both groups received prenatal care. The mode of delivery and the abortion rate in both groups were similar. Twenty-eight percent of women in the Pre-076 group had cesarean delivery compared to $33 \%$ in the Post-076 group A similar proportion of women in both groups had received a diagnosis of AIDS according to the CDC criteria [13].

Figure 1 summarizes the study cohort and the sterilization choices of the HIV-infected women. Of the fifty-seven women in the Pre-076 group forty-two (74\%) chose postpartum sterilization for their method of contraception after completion of their lattermost pregnancy at PMH, compared to only 15 of the $57(26 \%)$ choosing to maintain their fertility. In the Post-076 Group, less than half of the women (139 of 310, 45\%) chose sterilization as their method of contraception after pregnancy completion, compared to 171 of $310(55 \%)$ choosing to maintain fertility. This difference between the Pre-076 group and the Post-076 group was statistically significant with $p<0.001$.

We analyzed subgroups to evaluate if age or parity affects sterilization decisions. We divided age into three categories for comparison: women under the age of 21 years, women ages 21-34 years, and

Table I. Selected demographics.

\begin{tabular}{|c|c|c|c|}
\hline & $\begin{array}{c}\text { Pre- } 076 \\
n=57\end{array}$ & $\begin{array}{c}\text { Post-076 } \\
n=310\end{array}$ & $p$-value \\
\hline Age, yrs (mean $\pm S D$ ) & $25.9 \pm 9.0$ & $26.1 \pm 5.7$ & NS \\
\hline Race & & & NS \\
\hline Black & $40(70 \%)$ & $213(69 \%)$ & \\
\hline Hispanic & $9(16 \%)$ & $56(18 \%)$ & \\
\hline White & $7(12 \%)$ & $39(13 \%)$ & \\
\hline Other & $1(2 \%)$ & $1(<1 \%)$ & \\
\hline Type of Delivery & & & NS \\
\hline Vaginal Delivery & $34(60 \%)$ & $171(55 \%)$ & \\
\hline Cesarean Delivery & $16(28 \%)$ & $102(33 \%)$ & \\
\hline Abortion & $7(12 \%)$ & $37(12 \%)$ & \\
\hline Prior HIV-Infected Child & $5(9 \%)$ & $14(5 \%)$ & NS \\
\hline Date of HIV Diagnosis & & & NS \\
\hline Pre-Pregnancy & $26(46 \%)$ & $171(55 \%)$ & \\
\hline 1-42 Weeks Gestation & $30(53 \%)$ & $123(40 \%)$ & \\
\hline Postpartum & $1(2 \%)$ & $16(5 \%)$ & \\
\hline Diagnosed with AIDS & $9(16 \%)$ & $57(19 \%)$ & NS \\
\hline Received Prenatal Care & $52(91 \%)$ & $295(95 \%)$ & NS \\
\hline
\end{tabular}

Using Chi-sqare and Student's t-test, $\mathrm{p}<0.05$ is significant. NS, not significant

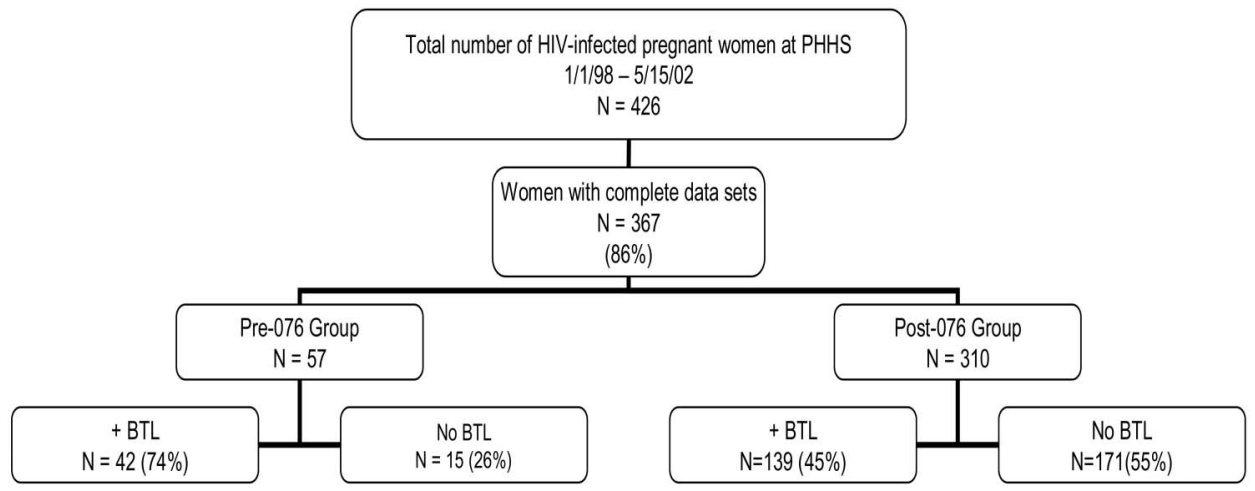

Figure 1. Sterilization choices of HIV-infected women who completed their last pregnancy at Parkland Memorial Hospital 1 January, 1993 31 December, 2002. 
women older than 34 (Table II). Amongst women younger than 21 in the Pre-076 Group, ten out of 14 $(71 \%)$ chose BTL, compared to 20 out of the 37 (35\%) women younger than 21 years in the Post-076 Group; this difference was significant with a $p$-value of 0.014 . Women between the ages of 21-34 also showed a significant decrease in their choice of sterilization with 28 of $39(72 \%)$ in the Pre-076 Group choosing sterilization compared to 101 of 225 $(45 \%)$ in the Post-076 Group, $p=0.002$. There was no significant difference between women older than 34 years in the two groups.

We divided parity into four groups to assess if parity had an effect on sterilization decisions: women who had one child, two children, three children, or more than three children (Table III). In the Pre-076 Group seven out of nine (78\%) primiparous women chose sterilization as their contraceptive method compared to 10 of 74 (14\%) in the Post-076 Group, $p<0.001$. A statistically significant difference was also seen among women who had delivered three babies; 16 of $18(88 \%)$ of these women chose sterilization in the Pre-076 Group compared to only 39 of $66(59 \%)$ in the Post-076 Group $p=0.018$.

Logistic regression was done to evaluate how choices for sterilization may have changed over time. We evaluated the probability of BTL by delivery date. This demonstrated a significant decrease over time in women choosing BTL, $p=0.002$. The choice for contraceptive method other than BTL was abstracted from the medical records and was therefore not available for every study subject. Over time, choice for condom use increased from $1 \%$ to $6 \%$ and choice for medroxyprogesterone-acetate increased from $2 \%$ to $12 \%(p<0.0001)$.

\section{Comment}

Our study indicates that significantly fewer HIVinfected women chose postpartum sterilization after it was known that medical intervention with zidovudine during pregnancy and the immediate postpartum period would decrease HIV vertical transmission. Seventy-four percent of HIV-infected women chose BTL prior to routine PACTG 076 implementation at our institution compared to only $45 \%$ in the Post076 Group. Additionally, over time, the probability of a woman choosing sterilization as her method of contraception significantly decreased. When age and parity were evaluated, fewer younger women and fewer primiparous women chose sterilization in more recent years.

With the advent of PACTG 076, regular use of triple antiviral therapy, and subsequent regular use of highly-active antiretroviral therapy (HAART) and cesarean delivery, the rate of maternal to child transmission has continued to decline. Throughout the era of HIV, pregnant women infected with HIV

Table II. The association of age with women who chose postpartum bilateral tubal ligation in the Pre-076 and Post-076 Groups.

\begin{tabular}{|c|c|c|c|c|c|}
\hline \multirow[b]{2}{*}{ Age (years) } & \multicolumn{2}{|c|}{ Pre-076 } & \multicolumn{2}{|c|}{ Post-076 } & \multirow[b]{2}{*}{$p$-value } \\
\hline & $\begin{array}{c}\text { Chose BTL } \\
N=42\end{array}$ & $\begin{array}{c}\text { Chose fertility } \\
\qquad N=15\end{array}$ & $\begin{array}{c}\text { Chose BTL } \\
N=139\end{array}$ & $\begin{array}{c}\text { Chose fertility } \\
\quad N=171\end{array}$ & \\
\hline & & & & & 0.0001 \\
\hline 21 & $10(71 \%)$ & $4(29 \%)$ & $20(35 \%)$ & $37(35 \%)$ & 0.0136 \\
\hline $21-34$ & $28(72 \%)$ & $11(28 \%)$ & $101(45 \%)$ & $124(55 \%)$ & 0.0019 \\
\hline$>34$ & $4(100 \%)$ & 0 & $18(64 \%)$ & $10(36 \%)$ & NS \\
\hline
\end{tabular}

Using Chi-sqare and Student's $t$-test, $p<0.05$ is significant. NS, not significant. ${ }^{\star}=$ total number of women of each age in each cohort. $21(N=14,57) ; 21-34(N=39,225) ;>34(N=4,28)$.

Table III. The association of parity and age with women who chose postpartum bilateral tubal ligation (PPBTL) in the Pre-076 compared to Post-076 Groups.

\begin{tabular}{|c|c|c|c|c|c|}
\hline \multirow[b]{2}{*}{ Parity^ } & \multicolumn{2}{|c|}{ Pre-076 } & \multicolumn{2}{|c|}{ Post-076 } & \multirow[b]{2}{*}{$p$-value } \\
\hline & $\begin{array}{c}\text { Chose BTL } \\
N=42\end{array}$ & $\begin{array}{c}\text { Chose Fertility } \\
\qquad N=15\end{array}$ & $\begin{array}{c}\text { Chose BTL } \\
N=139\end{array}$ & $\begin{array}{c}\text { Chose Fertility } \\
\qquad N=171\end{array}$ & \\
\hline & & & & & 0.0001 \\
\hline 1 & $7(78 \%)$ & $2(22 \%)$ & $10(14 \%)$ & $64(86 \%)$ & $<0.001$ \\
\hline 2 & $12(57 \%)$ & $9(43 \%)$ & $58(48 \%)$ & $63(52 \%)$ & NS \\
\hline 3 & $16(88 \%)$ & $2(12 \%)$ & $39(59 \%)$ & $27(41 \%)$ & 0.0184 \\
\hline$>3$ & $7(78 \%)$ & $2(12 \%)$ & $32(65 \%)$ & $17(35 \%)$ & NS \\
\hline
\end{tabular}

Using Chi-sqare and Student's $t$-test, $p<0.05$ is significant. NS, not significant. ${ }^{\star}=$ total number of women of each parity in each cohort. $1(N=9,74) ; 2(N=21,121) ; 3(N=18,66) ;>3(N=9,49)$. 
have received thorough counseling at our institution primarily by the same clinicians (G.W., J.S. and B.M.). HIV-infected women receive updated information regarding rates of HIV transmission risk and status of their own HIV disease throughout the counseling.

The choice for contraceptive method overall also changed significantly over time $(p<0.0001)$. This is attributable to the increase in condom use from $1 \%$ to $6 \%$ and the increase in Depo-Provera ${ }^{\circledR}$ from $2 \%$ to $12 \%$ in the Pre-076 Group compared to the Post076 Group. It is notable that in both groups the contraceptive method most commonly chosen was BTL. A high proportion of HIV-infected women in our cohort underwent postpartum sterilization despite non-judgmental and non-directional counseling. We suspect this was due to the poor prognosis of HIV disease at the time. We could find no law in the state of Texas prohibiting sterilization of women below a certain age, nor a law requiring a certain amount of patient contact prior to the procedure.

Our findings are consistent with other published studies. Investigators from Brazil found that knowledge of HIV seropositive status increased the prevalence of bilateral tubal ligation [12]. A study on the impact of the knowledge of HIV serostatus and contraceptive choice and repeat pregnancy found that seropositive women were less likely to have a subsequent pregnancy and more likely to choose sterilization [11]. This is in contrast to a cohort of Midwestern non-injecting drug use women in whom knowledge of HIV infection and the use of zidovudine to decrease vertical transmission was not associated with a change in abortion consideration or pregnancy planning [9]. A survey of 1,421 HIV-infected men and women [14] found that $69 \%$ of reproductive age HIV-infected women in the United States desire to have children in the future. We believe our finding that fewer young, nulliparous women are choosing BTL is due to the decreasing vertical transmission rates associated with the use of effective antiretroviral therapy. The status of HIV disease is one factor of many, including sociocultural and behavioral beliefs, which can impact reproductive decision making [15]. As indicated by our demographic data the basic demographics of our population were not different between the two groups; therefore, we believe the decrease in sterilization is largely due to the decrease in the rate of maternal to child transmission of HIV. Our study supports the argument that as HIV infection continues to change to a chronic asymptomatic disease HIV-infected women can look for a brighter future with more childbearing plans than they had in the past.

In this study postpartum sterilization has been used as a surrogate for measurement of fertility wishes in HIV-infected women. This can be misleading both because adequate reversible contraceptive options are available and because fertility in HIV-infected women can be affected by anovulation and infertility as a result of HIV-related illnesses [16]. Also, it is possible that some women who chose not to have a tubal ligation at PMH may have gone to another hospital for a subsequent pregnancy where they may have decided to have a tubal ligation. We believe that most HIV-infected women cared for at PMH chose to return to $\mathrm{PMH}$ for their subsequent pregnancyrelated care, as evidenced by the 78 women who had multiple pregnancies at PMH. Thus, loss to follow up is likely minimal. Abortions were not separated and analyzed differently because the cohort was small and we felt analysis of this subgroup would not add anything to the study.

There are changes over time which can affect a before and after study design such as this one. Specifically, there were changes in mandatory HIV testing of pregnant women during the study period which could affect HIV disease ascertainment. In 1996 it became mandatory for all pregnant women in the state of Texas to be offered HIV testing when they presented for initiation of prenatal care, and at time of labor and delivery [17]. It is therefore possible all HIV-infected pregnant women were not detected at PMH prior to 1996, although pregnant women who were considered to be high risk, due to a history of previous sexually transmitted infections or drug use, were counseled and offered HIV testing at our institution before mandatory testing was implemented in Texas.

With the use of cesarean delivery and additional anti-retroviral medications, a further reduction in the vertical transmission rate to less than $2 \%$ [2] has contributed to an even further decline than the initial decline in transmission rate from $28 \%$ to $8 \%$ after PACTG Protocol 076 was initiated. As life expectancy and disease manifestation of HIV-infection continues to improve due to continued advances in disease management, it is reasonable to expect that more HIV-infected women will want to maintain their options for future fertility. As shown in this report, fewer HIV-infected women are choosing sterilization and more HIV-infected women are choosing reversible methods of contraception. Our conclusions support the need for continuing research to address the special reproductive needs of HIVinfected women as we continue in the era of HIV as a chronic infection.

\section{References}

1. HIV/AIDS Surveillance in Women. L264 slide series through 2001, Slide 8 of 8 . Women: Minority and young women at continuing risk. Available: http://www.cdc.gov/hiv/graphics/ images/1264/1264-8.htm. Retrieved 7/20/04. 
2. The International Perinatal HIV Group. The mode of delivery and the risk of vertical transmission of human immunodeficiency virus type 1-a meta-analysis of 15 prospective cohort studies. N Engl J Med 1999;340:977-987.

3. Connor EM, Sperling RS, Gelber R, Kiselev P, Scott G, O’Sullivan MJ, Van Dyke R, Bey M, Shearer W, Jacobson RL. Reduction of maternal-infant transmission of human immunodeficiency virus type 1 with zidovudine treatment. Pediatric AIDS Clinical Trials Group Protocol 076 Study Group. N Engl J Med 1994;331:1173-1180.

4. Dorenbaum A, Cunningham CK, Gelber RD, Culnane M, Mofenson L, Britto P, Rekacewicz C, Newell ML, Delfraissy JL, Cunnningham-Schrader B, et al. Two-dose intrapartum/ newborn nevirapine and standard antiretroviral therapy to reduce perinatal HIV transmission: a randomized trial. JAMA 2002;288:189-198.

5. Public Health Service Task Force. Recommendations for Use of Antiretroviral Drugs in Pregnant Women Infected with HIV-1 for Maternal Health and for Reducing Perinatal HIV-1 Transmission in the United States. Nov 2002 Guidelines.

6. Palella FJ, Jr, Delaney KM, Moorman AC, Loveless MO, Fuhrer J, Satten GA, Aschman DJ, Holmberg SD. Declining morbidity and mortality among patients with advanced human immunodeficiency virus infection. HIV Outpatient Study Investigators. N Engl J Med 1998;338:853-860.

7. Sunderland A, Minkoff HL, Handte J, Moroso G, Landesman $S$. The impact of human immunodeficiency virus serostatus on reproductive decisions of women. Obstet Gynecol 1992; 79:1027-1031.

8. Thackway SV, Furner V, Mijch A, Cooper DA, Holland D, Martinez P, Shaw D, van Beek I, Wright E, Clezy K, et al. Fertility and reproductive choice in women with HIV-1 infection. AIDS 1997;11:663-667.
9. Smits AK, Goergen CA, Delaney JA, Williamson C, Mundy LM, Fraser VJ. Contraceptive use and pregnancy decision making among women with HIV. AIDS Patient Care STDS. 1999;13:739-746.

10. Stephenson JM, Griffioen A. The effect of HIV diagnosis on reproductive experience. Study Group for the Medical Research Council Collaborative Study of Women with HIV. AIDS 1996;10:1683-1687.

11. Lindsay MK, Grant J, Peterson HB, Willis S, Nelson P, Klein L. The impact of knowledge of human immunodeficiency virus serostatus on contraceptive choice and repeat pregnancy. Obstet Gynecol 1995;85:675-679.

12. Magalhaes J, Amaral E, Giraldo PC, Simoes JA. HIV infection in women: impact on contraception. Contraception 2002;66: 87-91.

13. CDC. 1993 Revised classification system for HIV infection and expanded surveillance case definition for AIDS among adolescents and adults. MMWR 1993;41 (No. RR-17).

14. Chen JL, Philips KA, Kanouse DE, Collins RL, Miu A. Fertility desires and intentions of HIV-positive men and women. Fam Plann Perspect 2001;33:144-52, 165.

15. Selwyn PA, Carter RJ, Schoenbaum EE, Robertson VJ, Klein RS, Rogers MF. Knowledge of HIV antibody status and decisions to continue or terminate pregnancy among intravenous drug users. JAMA 1989;261:3567-3571.

16. Ross A, Morgan D, Lubega R, Carpenter LM, Mayanja B, Whitworth JA. Reduced fertility associated with HIV: the contribution of pre-existing subfertility. AIDS 1999, 13:21332141.

17. Grimes RM, Helfgott AW, Watson JR, Eriksen NL. For children's sake. New law mandates HIV testing of pregnant patients. Tex Med 1996;92:36-40. 


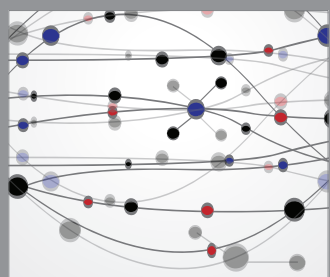

The Scientific World Journal
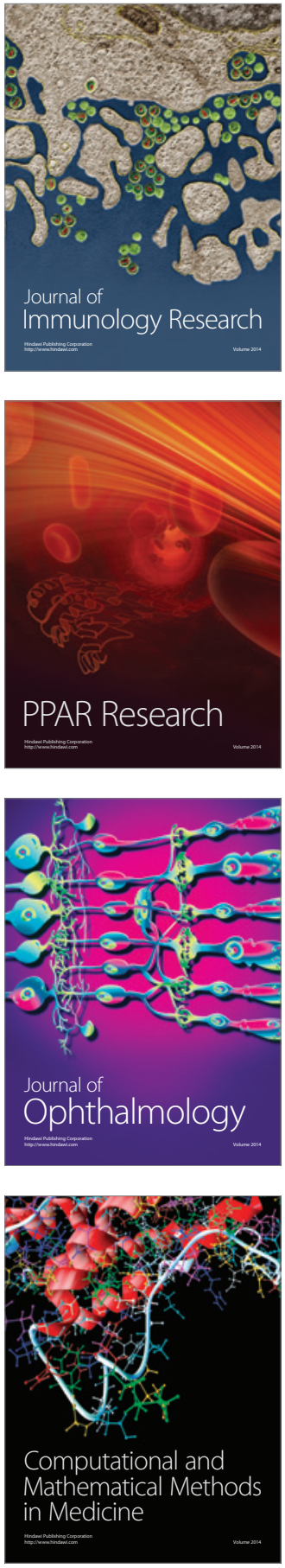

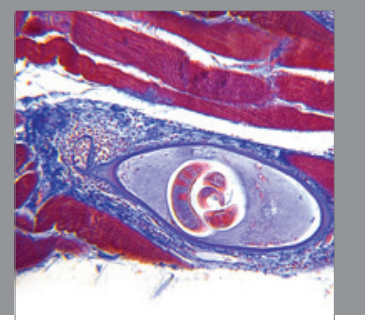

Gastroenterology

Research and Practice
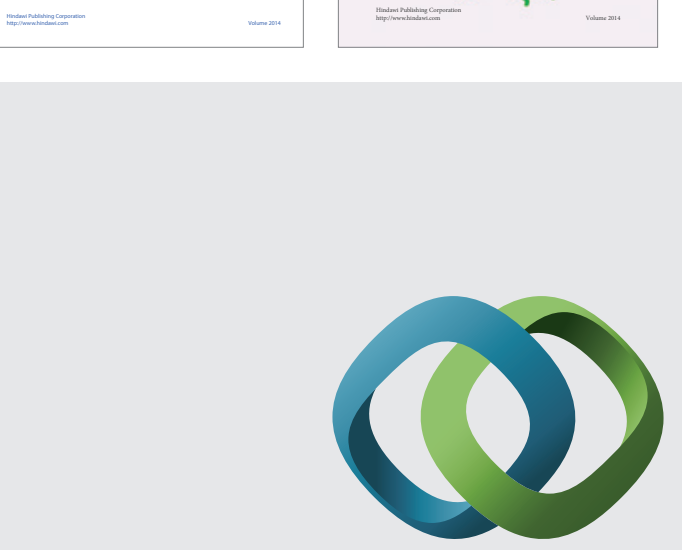

\section{Hindawi}

Submit your manuscripts at

http://www.hindawi.com
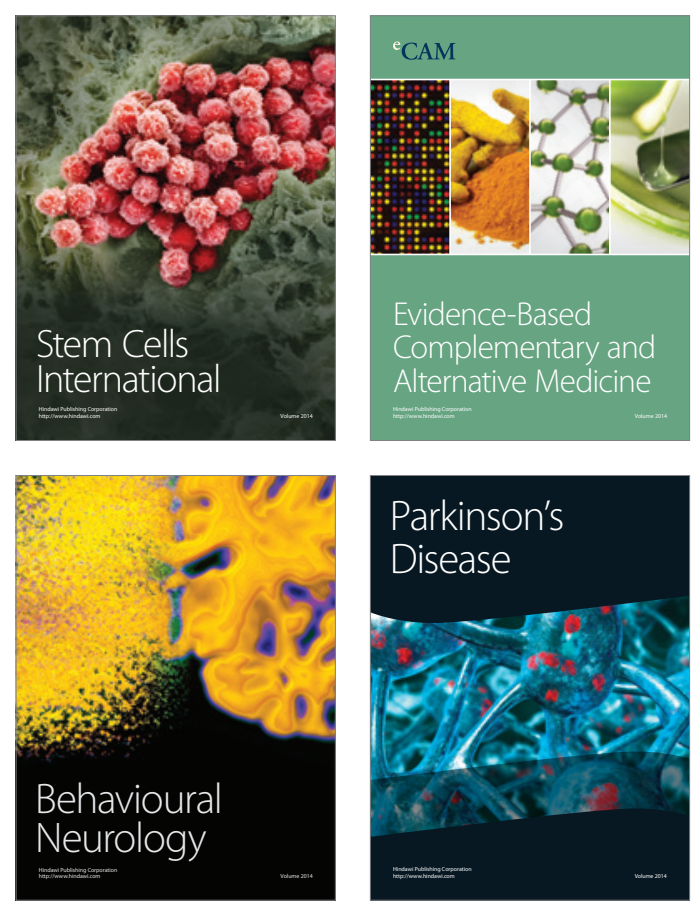

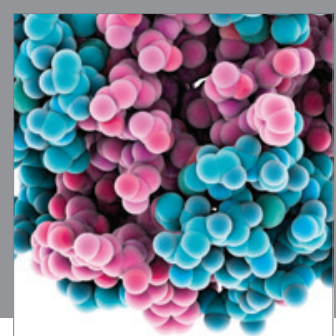

Journal of
Diabetes Research

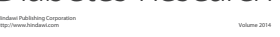

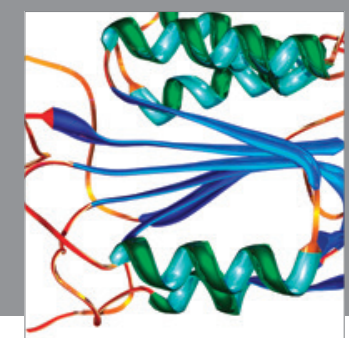

Disease Markers
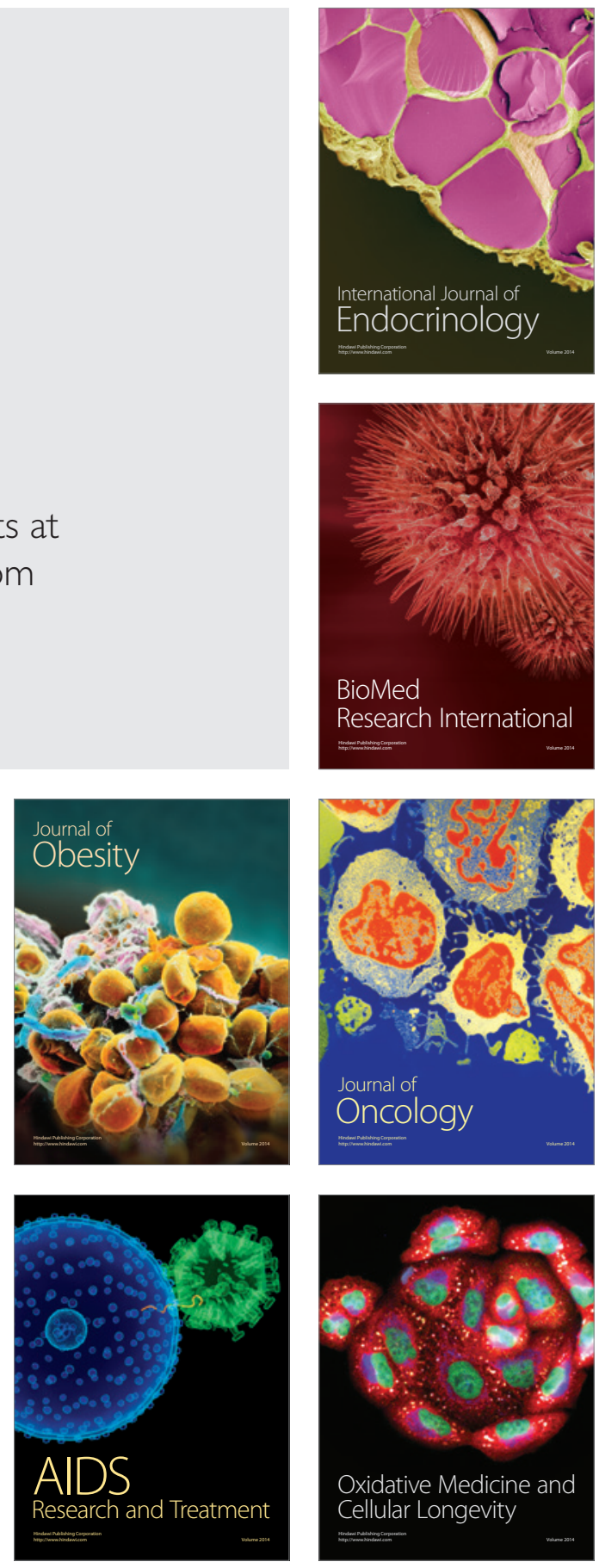